\title{
34 Year Old Woman With Thrombocytopenia and Weakness
}

\author{
M. Paula Martinez, MD and Ubaldo E. Martinez, MD
}

Patient is a 34 year old Liberian Female with past medical history significant for Systemic Lupus Erythematosus (SLE) that was recently admitted to the hospital for weakness and was diagnosed with encephalomyelitis due to SLE. At that time the patient was also noted to have low platelets felt secondary to Immune Thrombocytopenic Purpura (ITP). She has history of thrombocytopenia with her four pregnancies. During this past admission the patient was treated with hydroxychloroquine and high dose steroids for the CNS involvement of SLE but no platelet response was noted with administration of steroids.

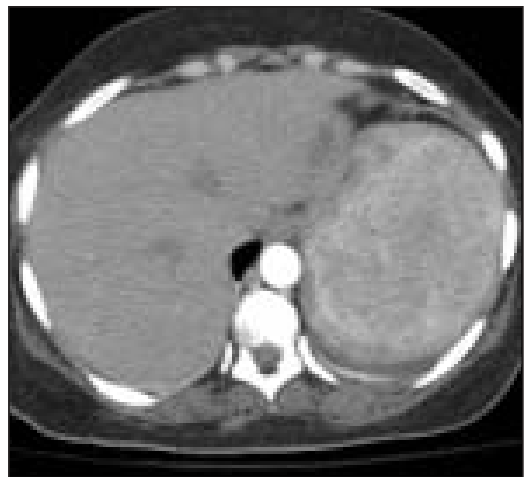

Figure 1. CT angiogram of chest with finding of large splenic mass. reticulocyte 2.2\%, LDH $188 \mathrm{IU} / \mathrm{L}$, haptoglobin $115 \mathrm{mg} / \mathrm{dL}$, total bilirrubin 0.5 $\mathrm{mg} / \mathrm{dL}$, creatinine $0.5 \mathrm{mg} / \mathrm{dL}$. INR $1.21, \mathrm{PT}$ 15.8 sec, PTT 27 sec. Fibrinogen 480 $\mathrm{mg} / \mathrm{dL}$, D-Dimer $6.49 \mathrm{mcg} / \mathrm{ml}$. Peripheral smear showed decreased platelets without clumping and scarce schistocytes.

The patient's blood smear was not consistent with TTP. ITP was felt to be the most likely cause of her thrombocytopenia and she underwent treatment initially with high dose steroids and subsequently with IVIG, both without significant response. Her bone marrow biopsy showed hyperplastic megakaryocytosis consistent with peripheral destruction. She also underwent treatment with rituximab for encephalomyelitis without platelet response. A CT Angiogram of chest was ordered during her hospitalization for an episode of shortness of breath. There was no evidence of pulmonary embolism but a large heterogeneous splenic mass was visualized (Figure 1). This mass upon further evaluation with MRI was hypervascular and the differential diagnosis included angiosarcoma and hemangioma.

The patient underwent splenectomy and pathology revealed a large splenic hemangioma with necrosis. Platelet counts steadily rose from the day of splenectomy and remained stable during the remainder of her hospitalization.

\section{Discussion}

This patient had thrombocytopenia from splenic sequestration due to a large hemangioma. Hemangiomas are the most common primary tumor of the spleen with a prevalence of $0.03-0.14 \%$. This is a congenital lesion arising from sinusoidal epithelium resulting from proliferation of vascular channels lined by a single layer of endothelium, most often resulting in a cavernous lesion.

Hemagiomas are variable in size ranging from a few millimeters to several centimeters. The majority of these tumors are less than $4 \mathrm{~cm}$, but there are reports of lesions up to $17 \mathrm{~cm}$ in diameter. Hemangiomas may be single or multiple as in Klippel-TrenauneyWeber Syndrome.

The majority of hemangiomas are asymptomatic and incidentally discovered. However, larger lesions may enlarge the spleen leading to fullness and left upper quadrant discomfort, spontaneous splenic rupture or Kasabach-Merritt phenomenon (thrombocytopenia and/or coagulopathy, now called disseminated intravascular coagulation or DIC, that results from platelet trapping within a vascular tumor). Thrombocytopenia results from shortened platelet survival caused by sequestration of platelets in the vascular malformation. Episodes of acute DIC have been reported in pregnant women with congenital hemangiomata and in one woman during two successive pregnancies. The hormonal

$\mathrm{g} / \mathrm{dL}$, platelets $57000 / \mathrm{microL}, \mathrm{MCV}$ 70fl, RDW $21.7 \%$, 


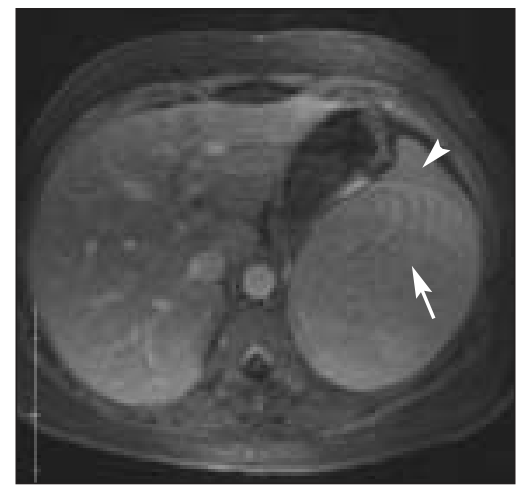

Figure 2. T1 weighted MRI revealing large splenic mass (arrow) that is hypervascular compared to normal splenic tissue (arrowhead). alterations and increase in blood volume in pregnancy may affect pre-existing lesions, triggering episodes of acute DIC.

This patient presented with an uncommon cause of thrombocytopenia in the general population. Our approach to the patient with decreased platelets should first be focused on ruling out potential fatal causes such as TTP with the peripheral smear and pseudothrombocytopenia or clumping. In the differential diagnosis of low platelet counts one should also include medications, alcohol abuse, viral infection, hypersplenism, disseminated intravascular coagulation, myelodysplastic syndrome. ITP should be considered as a diagnosis of exclusion. Common medications associated with thrombocytopenia include heparin, glycoprotein IIb/IIIa inhibitors, diuretics, trimethoprim-sulfamethoxazole and quinine.

\section{References}

1. Hall GW. Kasabach-merritt syndrome: pathogenesis and management. Br J Haematol 2001; 112(4-II): 851-862.

2. Kutok JL, Fletcher CD. Splenic vascular tumors. Semin Diagn Pathol 2003; 20(2):128-39.

3. Kaido T, Imamura M. Giant hepatic hemangioma. N Eng Med 2003; 349(20): e 19.

\title{
Case Reports
}

\section{Liver Transplant Recipient With Bilateral Leg Swelling}

\author{
Kuntal M. Thaker, MD, Maya Spodik, MD, Kuldip S. Banwait, MD, Steven K. Herrine, MD, and Victor Navarro, MD
}

A 50-year-old white male was admitted to the hospital with erythema and swelling of both lower extremities.

The patient had a medical history significant for liver transplant five years prior for end-stage liver disease secondary to alcoholic cirrhosis. Following transplant, he developed mitral valve insufficiency with heart failure and pulmonary hypertension. He had been in his usual state of health until approximately a week prior to admission when he noticed that his lower extremities had increased in girth. For several days, he noted that there had also been increased redness and pain over the affected area.

The patient denied other symptoms such as headache, photophobia, phonophobia, or weakness. He had no report of dyspnea or a new productive cough. There was no other significant past medical or surgical history. Prior to his initial diagnosis, his alcohol consumption was difficult to quantify, however, since several years prior to transplant, the patient had remained abstinent. He had not traveled outside of the United States. His immunosuppressive regimen consisted of prednisone $10 \mathrm{mg}$ and sirolimus $1 \mathrm{mg}$ once a day and knew of no allergies to medications.

The vital signs were significant only for a temperature of $100.8^{\circ} \mathrm{F}$; all other vital signs were normal.

On physical examination, the head, neck, heart, lungs, and abdomen were normal. Multiple, irregularly-shaped, deep ulcerations with surrounding erythema were seen on both lower extremities. There was no evidence of necrosis, however, there was significant edema of the lower extremities.

The patient was started on a regimen of broad-spectrum antibiotics consisting of intravenous vancomycin and piperacillin/tazobactam for the treatment of cellulitis in an immunocompromised host. Initially, there was no resolution of the cellulitis or ulceration. Blood cultures remained persistently negative. Magnetic resonance imaging of the lower extremities revealed cellulites of the right lower extremity and myositis with fasciitis on the left.

Biopsy of the skin lesions revealed diffuse yeast forms with mucicarmine positive capsules consistent with cryptococcus infection. Cryptococcal antigen titer was markedly elevated at 1:251, consistent with cryptococcus infection. Evaluation of cerebrospinal fluid did not reveal meningeal involvement. The patient was started on amphotericin B with complete resolution of the lesions.

Cutaneous involvement is an uncommon manifestation of cryptococcal disease, but it may be the initial manifestation of systemic cryptococcosis in solid-organ transplant (SOT) recipients and other immunocompromised hosts. Only 36 cases have been described in the literature and majority of them are in renal transplant recipients. No cases of cellulitis with fasciitis and myositis without systemic involvement have been described for liver transplant patients. We describe a liver transplant recipient diagnosed with cellulites, fasciitis, and myositis as the only presenting manifestation of cryptococcal infection..

Cryptococcal infection must be included in the differential diagnosis of cellulitis in SOT recipients. Deep soft tissue involvement may occur concomitantly with primary cutaneous cryptococcosis. Because the clinical appearance of cutaneous cryptococcosis is non-specific, early diagnosis requires tissue acquisition for testing. Tissue samples should be stained with the fungal stain mucicarmine to reveal the characteristic organisms. Cryptococcal antigen test is a simple blood test that may aid in the diagnosis of cryptococcosis. 